\title{
IL-37 protects myocardial ischemia reperfusion injury in mice through mediating inflammation response.
}

\author{
Huaping Xiao" ${ }^{1}$, Bingda $\mathrm{Li}^{1}$, Xiaomin Yang${ }^{1}$, Qiulin Yin ${ }^{2 *}$ \\ ${ }^{1}$ Department of Anaesthesia, Jiangxi Cancer Hospital, Nanchang, Jiangxi, PR China \\ ${ }^{2}$ Department of Cardiology, Jiangxi Provincial People's Hospital, Nanchang, Jiangxi, PR China
}

\begin{abstract}
Objective: To investigate the mechanism of IL-37 in alleviating myocardial ischemia reperfusion injury by regulating inflammatory response balance.

Method: 30 Wistar male mice were randomly and equally assigned into Sham group (Sham operation), IL-37 intervention group (I/R plus IL-37 intervention, short for experimental group) and control intervention group ( $/ / R+$ normal saline, short for control group), respectively. Sham operation or myocardial Ischemia Reperfusion ( $\mathbf{I} / \mathbf{R})$ model were performed in Wistar male mice. Mice in experimental group received human recombined IL-37 intervention. The infarction area and cardiac function were examined. The expression of TNF- $\alpha$, IL-1 $\beta$, IL- 6 and TGF- $\beta$ in serum and myocardial infarction border zone were measured. MPO activity was examined as an indicator of neutrophil recruitment.

Result: Compared with control group, $\mathbf{I} / \mathbf{R}$ infarction area of mice in experimental group were significantly decreased $(P<0.01)$, while EF and FS was increased remarkably $(P<0.05)$. Compared with Sham group, the level of TNF- $\alpha$, IL-1 $\beta$, IL- 6 and TGF- $\beta$ in serum and myocardial infarction border zone was increased both in control and experimental group $(P<0.01)$, with significantly increased MPO activity $(\mathbf{P}<0.01)$. Moreover, the level of TNF- $\alpha$, IL-1 $\beta$, IL-6 and MPO in serum and myocardial infarction border zone in experimental group was significantly lower than that in control group $(\mathbf{P}<0.01)$. However, the TGF- $\beta$ level was higher in experimental group than that in control group $(\mathbf{P}<0.01)$.

Conclusions: IL-37 could decrease I/R infarction area and improve cardiac function after myocardial ischemia reperfusion injury. The protective effect of IL-37 against I/R injury might be associated with down-regulating the secretion of proinflammatory factors, inhibiting MPO expression and up-regulating the secretion of anti-inflammatory cytokines.
\end{abstract}

Keywords: IL-37, Ischemia reperfusion injury, Inflammatory response, Neutrophil.

Accepted on November 15, 2017

\section{Introduction}

Myocardial Ischemia Reperfusion (I/R) injury is defined as aggravated cardiac damage occurring in recovering of blood flow after myocardial ischemia, and such injury always leads to development of heart failure [1]. One of pathogenic mechanism for I/R injury is innate immunity and inflammatory responses which are activated by myocardial necrotic material and deteriorated extracellular matrix via TLRs [2]. Researchers found activated TLR4/NF- $\kappa \mathrm{B}$ signaling led to increased expression of inflammatory cytokines and chemokines after myocardial ischemia, followed by production of proteolytic enzymes and oxygen free radicals during inflammatory cells recruitment, thus myocardial cell injury was exacerbated and ischemia tolerance of myocardial cell deteriorated, leading to impaired cardiac function [3].
IL-37 is a member of IL-1 family and widely distributed in various tissues. As a natural inhibitor of innate immune, IL-37 is not only involved in the regulation of inflammation factors, but also participates in autoimmune disease and immunoreaction of inflammatory diseases, including ischemiareperfusion disease of alimentary system [4]. A previous study demonstrated that IL-37 inhibited Toll-like Receptor (TLR)-4 expression and nuclear factor kappa B (NF-kB) activation after $\mathrm{I} / \mathrm{R}$, while increasing the anti-inflammatory IL-10 level [5]. In addition, it was proved that IL-37 is expressed in the foam cells of coronary atherosclerosis plaque, but there was still lacking of related clinical trials [6]. It was proved that mice could not produce and express IL-37, so we planned to use human recombined IL-37 to replenish IL-37 in mice. 


\section{Materials and Methods}

\section{Experimental animals}

The $8-10$ w 80 cleaning laboratory Wistar male mice with a weight of 50-70 $\mathrm{g}$ were purchased from experimental animal center of Nanchang University, license number: SCXK (Zhe) 2015-0004. Those mice were kept in separate cages with the temperature ranging from $20-25^{\circ} \mathrm{C}$ and air relative humidity from $50-60 \%$.

Mice were used for all experiments, and all procedures were approved by the Animal Ethics Committee of Jiangxi Cancer Hospital.

\section{Experimental materials}

Ketamine and diazepam were respectively purchased from Shanghai Boulder biotechnology Company and Nantong Jinhua Pharmaceutical Group Company (approved by H32020869); Elisa assay kits for measuring TNF- $\alpha$, IL-1 $\beta$, IL-6, and TGF- $\beta 1$ were from Shanghai Yanjing biotechnology company. TNF- $\alpha$, IL- $1 \beta$, IL-6, and TGF- $\beta 1$ rodent ventilator were from Shanghai Kemin biotechnology company; the constant temperature shaker were provided by Zhejiang Chinese Medical University Laboratory Animal Research Center.

\section{Experimental methods}

Animal model and grouping method: Animal models were performed based on a previous study [7]. Briefly, after anaesthetization through intraperitoneal injection of $75 \mathrm{mg} / \mathrm{kg}$ Ketamine and $7.5 \mathrm{mg} / \mathrm{kg}$ diazepam, the oral endotracheal intubation was provided for assisted respiration of mouse on the plat, then opened the chest along with the midline for uncovering the heart, the left anterior descending branch of experimental group was ligated by 6-0 mersilk within $30 \mathrm{~min}$, those successfully modelled 20 mice were allocated randomly: IL-37 treatment (experimental group), saline control (control group), 10 mice in each group, the chest of other 10 sham mice were only opened and uncovered, but not ligation. $1 \mu \mathrm{g}$ human recombinant IL-37 (dissolution in $200 \mu \mathrm{L} 0.9 \% \mathrm{NaCl}$ ) was tail vein injected into the experimental group when reperfusion, instead, the control were tail vein injected with $200 \mu \mathrm{L} 0.9 \%$ $\mathrm{NaCl}$. Mice in each group were sacrificed after $24 \mathrm{~h}$.

\section{Detection index}

Myocardial ischemic necrosis area: Echocardiography was performed after myocardial ischemia reperfusion, and Ejection Fraction (EF) and short axial shortening rate (FS) were detected. Mice were sacrificed after detection to stop the heartbeat in ventricular diastolic stage, opened the chest, uncovered the heart and removed the residual blood, ligated the left anterior descending branch again, injected $1 \mathrm{ml} 1 \%$ EVANS along mouse aorta into chambers of the heart, the nonischemic area was dyed blue, while ischemic area undyed, cut the heart into $1 \mathrm{~mm}$ section and placed into $1 \%$ TTC solution, incubated in $37^{\circ} \mathrm{C}$ for $20 \mathrm{~min}$, the myocardial ischemic area was dyed blue, however, it was white in the myocardial infarction area, the area of relative ischemic dyed red. Area of myocardial ischemia and infarction were detected by Image Pro Plus software, the size of myocardial ischemic area=weight of myocardial ischemic area/weight of left ventricle, size of myocardial infarction=weight of myocardial infarction/weight of myocardial ischemia.

\section{Detection of serum TNF- $\alpha, I L-1 \beta, I L-6$ and TGF- $\beta$}

After euthanasia, the myocardium was cleared with $0.9 \% \mathrm{Nacl}$, and part of ischemic myocardium was made into $10 \%$ myocardial homogenate, in which the MPO neutrophil recruitment (MPO) activity was detected by MPO kit according to manufacturer's instructions.

\section{Statistical analysis}

All test results were summarized and analysed by SPSS20.0 software. The results were shown as mean value \pm Standard Deviation (SD) and analysed by student-t test. $\mathrm{P}$ value $<0.05$ was considered to be statistically significant.

\section{Result}

\section{Analysis of myocardial ischemia reperfusion injury area}

According to experiment results, there was no significant ischemia in Sham group. Meanwhile, there was also no significant difference in ischemia area between experimental group and control group $(\mathrm{P}>0.05)$. However, the infarction size of mice from experimental group was significantly decreased compared with control group $(\mathrm{P}<0.01)$. All data was showed in Figure 1.

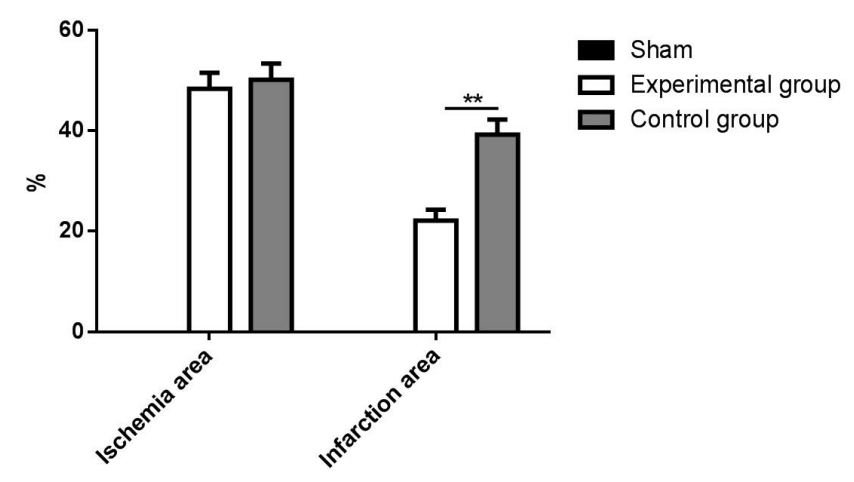

Figure 1. Comparison of ischemia and infarction area (\%) between control group and experimental group. ${ }^{* *} P<0.01$.

\section{Analysis of cardiac function}

Compared with Sham group, EF and FS was decreased significantly both in experimental group and control group $(\mathrm{P}<0.05)$. Moreover, EF and FS in experimental group was higher than that in control group $(\mathrm{P}<0.05)$. All data was showed in Figure 2. 


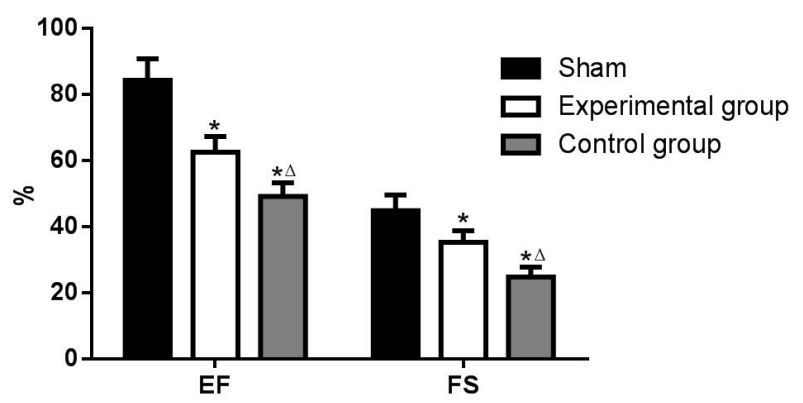

Figure 2. Comparison of cardiac function, including EF and FS (\%). Compared with Sham group, ${ }^{*} P<0.05$. Compared with experimental group, ${ }^{\Delta} P<0.05$.

\section{MPO activity and inflammatory factor expression}

Compared with Sham group, TNF- $\alpha$, IL-1 $\beta$, IL-6 and TGF- $\beta$ level in serum was increased significantly both in experimental group and control group $(\mathrm{P}<0.01)$ and they were also increased in ischemia myocardium $(\mathrm{P}<0.01)$. Compared with Sham group, MPO activity of myocardium in experimental group and control group was increased $(\mathrm{P}<0.01)$. Compared with control group, TNF- $\alpha$, IL-1 $\beta$ and IL-6 in experimental group was decreased both in the level of serum and ischemia myocardium $(\mathrm{P}<0.01)$. Notably, TGF- $\beta$ in experimental group was higher than control group, both in the level of serum and ischemia myocardium $(\mathrm{P}<0.01)$. All data was showed in Tables 1 and 2 .

Table 1. Comparison of inflammatory factor expression in serum. Compared with Sham group, ${ }^{*} P<0.01$. Compared with control group, ${ }^{\Delta} P<0.01$.

\begin{tabular}{lllll}
\hline Group & Sample & TNF- $\boldsymbol{\alpha}$ & IL-1 $\boldsymbol{\beta}$ & IL- 6 \\
\hline Sham & 10 & $37.85 \pm 3.54$ & $18.44 \pm 3.14$ & $124.74 \pm 21.47$ \\
\hline Experimental & 10 & $105.41 \pm 21.47^{*} \Delta$ & $68.24 \pm 14.78^{*} \Delta$ & $214.11 \pm 31.54^{*} \Delta$ \\
\hline Control & 10 & $229.74 \pm 30.19^{*} \Delta$ & $110.47 \pm 21.21^{*} \Delta$ & $41.21 \pm 6.21^{*} \Delta$ \\
\hline
\end{tabular}

Table 2. Comparison of inflammatory factor expression in ischemia myocardium and comparison of MPO in myocardium. Compared with Sham group, ${ }^{*} P<0.01$. Compared with control group, ${ }^{\Delta} P<0.01$.

\begin{tabular}{lllllll}
\hline Group & Sample & TNF- $\alpha$ & IL-1 $\beta$ & IL-6 & TGF- $\beta$ & MPO \\
\hline Sham & 10 & 1 & 1 & 1 & 1 & $0.17 \pm 0.07$ \\
\hline Experimental & 10 & $3.53 \pm 1.02^{* \Delta}$ & $2.17 \pm 0.98^{* \Delta}$ & $2.14 \pm 0.78^{* \Delta}$ & $4.18 \pm 1.07^{* \Delta}$ & $0.48 \pm 0.21^{* \Delta}$ \\
\hline Control & 10 & $6.42 \pm 1.14^{* \Delta}$ & $5.14 \pm 1.01^{* \Delta}$ & $4.78 \pm 1.04^{* \Delta}$ & $2.24 \pm 0.98^{* \Delta}$ & $0.98 \pm 0.47^{* \Delta}$ \\
\hline
\end{tabular}

\section{Discussion}

In recent years, there were many studies focusing on the pathological mechanism of myocardial ischemia reperfusion injury, with well-established mechanism including oxygen free radical injury and calcium overload [8]. Researchers found dynamic changes of inflammatory factors had positive correlation with post-ischemia myocardial necrocytosis and impaired cardiac functions, suggesting inflammatory response might be involved in the pathological mechanism of myocardial ischemia reperfusion injury [9]. Studies showed TLR4 could activate inherent immune and inflammatory response in the progress of myocardial $\mathrm{I} / \mathrm{R}$ injury, more specific, TLR4 could up-regulate the secretion of proinflammatory factors such as IL-1 $\beta$, TNF- $\alpha$ and IL-6 via activating downstream signaling factors, such as NF- $\mathrm{NB}$ or P38, leading to release of proteolytic enzyme and oxygen free radicals which exaggerate inflammatory response and myocardial I/R injury [10]. IL-37 was proved to inhibit the secretions of inflammatory factors activated by LPS, including IL-1 $\beta$, TNF- $\alpha$ and IL-6 [11]. IL-37 is widely distributed in heart, brain, kidney and bone marrow. In normal condition, IL-37 is at low concentration in monocytes, macrophages, NK cells and endothelial cells, while IL-37 is up-regulated after inflammatory response as well as TGF- $\beta$, suggesting IL-37 could inhibit expression of inflammatory factors and attenuate inflammatory response [12-14]. Clinical trials indicated IL-37 play a role in inhibiting the expression of inflammatory factors in allergic skin disease and autoimmune disease. IL-37 upregulated anti-inflammatory factor IL-10 and protected organisms in DSS-induced colitis model of rat [15]. Nolebeg et al. showed in the LPS-induced infection model of mice, IL-37 inhibited inflammatory response via binding to extracellular SIGIRR and repressed expression of inflammatory factors. This indicated that IL-37 might be associated with both extracellular and intracellular inflammation inhibition [16]. Our study showed IL-37 not only decreased the expression of proinflammatory factors, but also increased the expression of anti-inflammatory factor TGF- $\beta$. Previous animal studies showed IL-37 could inhibit the expression of proinflammatory factor M1 in macrophage, repress maturation of dendritic cells and increase the expression of TGF- $\beta$ via negative feedback of inflammatory factor inhibition, but detailed mechanism remains unclear $[17,18]$.

According to our study, MPO of ischemia myocardium in experimental group was decreased significantly, and our study indicated IL-37 attenuated neutrophil recruitment in ischemia myocardium, which was consistent with a previous study 
showing IL-37 inhibited neutrophil recruitment to colonic lamina propria [19]. Previous studies showed both SIGIRR and Smad3 could restrain neutrophil infiltration in the process of myocardial I/R injury. Studies on liver I/R injury also showed IL-37 could decrease the expression of inflammatory factors (KC and MIP-2) and inhibit neutrophil recruitment [20]. However, the mechanism of IL-37 in inhibiting effect to neutrophil recruitment was not fully understood. Our study provided preliminary evidence that IL-37 inhibited the activity of neutrophil via influencing the expression of neutrophil specificity chemotactic factor [21].

In conclusion, IL-37 improved cardiac function, decreased infarction area and played a protective role in the process of $\mathrm{I} / \mathrm{R}$ injury, which might by through inhibiting the expression of inflammatory factors and up-regulating the expression of antiinflammatory cytokine.

\section{Acknowledgments}

This work was supported by Jiangxi science and technology plan project (No. 20151BBG70138).

\section{References}

1. Garlanda C. The interleukin-1 family: back to the future. Immunity 2013; 39: 1003-1018.

2. Conti P. IL-37 a new IL-1 family member emerges as a key suppressor of asthma mediated by mast cells. Immunol Invest 2017; 46: 239-250.

3. Youssef R. Peroxisome proliferator-activated receptor gamma, a possible culprit in mycosis fungoides: an immunohistochemical study. J Eur Acad Dermatol Venereol 2012; 26: 1522-1532

4. Sharma S. The il-1 family member $7 \mathrm{~b}$ translocates to the nucleus and down-regulates proinflammatory cytokines. J Immunol 2008; 180: 5477-5482

5. Wu B. Interleukin-37 ameliorates myocardial ischaemia/ reperfusion injury in mice. Clin Exp Immunol 2014; 176: 438-451.

6. McNamee EN. Interleukin 37 expression protects mice from colitis. Proc Nat Acad Sci USA 2011; 108: 16711-16716

7. Sakai N. Interleukin-37 reduces liver inflammatory injury via effects on hepatocytes and non-parenchymal cells. J Gastroenterol Hepatol 2012; 27: 1609-1616.

8. Song L. Glucocorticoid regulates interleukin-37 in systemic lupus erythematosus. J Clin Immunol 2013; 33: 111-117.

9. Cottle DL. Fetal inhibition of inflammation improves disease phenotypes in harlequin ichthyosis. Human Mol Gene 2015; 24: 436-449.
10. Taleb S, Tedgui A, Mallat Z. Adaptive T cell immune responses and atherogenesis. Curr Opin Pharmacol 2010; 10: 197-202.

11. Gautier EL. Conventional dendritic cells at the crossroads between immunity and cholesterol homeostasis in atherosclerosis. Circulation 2009; 119: 2367-2375.

12. Gao W. Innate immunity mediated by the cytokine il-1 homologue 4 (il-1h4/il-1f7) induces il-12-dependent adaptive and profound antitumor immunity. J Immunol 2003; 170: 107-113.

13. Komarova Y, Malik AB. Regulation of endothelial permeability via paracellular and transcellular transport pathways. Ann Rev Physiol 2010; 72: 463-493.

14. Prondzinsky R. Acute myocardial infarction and cardiogenic shock: Prognostic impact of cytokines: Infgamma, tnf-alpha, mip-1beta, g-csf, and mcp-1beta. Medizinische Klinik, Intensivmedizin und Notfallmedizin 2012; 107: 476-484.

15. Chen TH. Left-right symmetry breaking in tissue morphogenesis via cytoskeletal mechanics. Circ Res 2012; 110: 551-559.

16. Ridker PM. Elevation of tumor necrosis factor-alpha and increased risk of recurrent coronary events after myocardial infarction. Circulation 2000; 101: 2149-2153.

17. Cesari M. Inflammatory markers and physical performance in older persons: The inchianti study. J Gerontol Biol Sci Med Sci 2004; 59: 242-248.

18. Tiainen K. Associations between inflammatory markers, candidate polymorphisms and physical performance in older Danish twins. Exp Gerontol 2012; 47: 109-115.

19. Van Brussel I. Potential use of dendritic cells for antiatherosclerotic therapy. Curr Pharm Desig 2013; 19: 5873-5882.

20. $\mathrm{Wu}$ BW. The potential role of il-37 in atherosclerosis. Die Pharmazie 2013; 68: 857-860.

21. Subramanian M. Treg-mediated suppression of atherosclerosis requires myd88 signaling in DCS. J Clinic Investig 2013; 123: 179-188.

\section{*Correspondence to}

Qiulin Yin

Department of Cardiology

Jiangxi Provincial People's Hospital

PR China 